\title{
Bobble-head doll syndrome: report of 2 cases and a review of the literature, with video documentation of the clinical phenomenon
}

\author{
Bryan Renne, PhD, ${ }^{1}$ Stefan Rueckriegel, MD, ${ }^{2}$ Sudheesh Ramachandran, MCh, ${ }^{1}$ \\ Julia Radic, MD, MPH, FRCSC, ${ }^{1}$ Paul Steinbok, MBBS, FRCSC, ${ }^{1}$ and \\ Ash Singhal, MD, MSc, FRCSC ${ }^{1}$
}

${ }^{1}$ Faculty of Medicine and the Division of Neurosurgery, University of British Columbia and British Columbia Children's Hospital, Vancouver, British Columbia, Canada; and 2Department of Neurosurgery, University Hospital Würzburg, Germany

\begin{abstract}
Bobble-head doll syndrome (BHDS) is a rare pediatric movement disorder presenting with involuntary 2- to 3-Hz head movements. Common signs and symptoms also found on presentation include macrocephaly, ataxia, developmental delay, optic disc pallor or atrophy, hyperreflexia, tremor, obesity, endocrinopathy, visual disturbance or impairment, headache, and vomiting, among others. The syndrome is associated with suprasellar cysts, third ventricular cysts, or aqueductal obstruction, along with a few other less common conditions. The cause of involuntary head motions is not understood. Treatment is surgical. The authors present 2 cases of BHDS. The first is a 14-year-old boy with BHDS associated with aqueductal obstruction and triventricular hydrocephalus secondary to a tectal tumor. He was successfully treated by endoscopic third ventriculostomy, and all symptoms resolved immediately in the recovery room. This case is unusual in its late age of symptom onset, the primacy of lateral ("no-no") involuntary head rotations, and the associated tectal tumor. The second case is a 7.5-year-old girl with BHDS associated with a suprasellar cyst. She was successfully treated with an endoscopic fenestration but preexisting endocrinopathy persisted, and the patient was diagnosed with autism spectrum disorder at age 12 years. This second case is more typical of BHDS. A comprehensive and up-to-date review of the literature of BHDS and video documentation of the phenomenon are presented.
\end{abstract}

https://thejns.org/doi/abs/10.3171/2017.9.PEDS16704

KEY WORDS bobble-head doll syndrome; head bobbing; aqueductal obstruction; tectal tumor; suprasellar cyst; oncology

$\mathrm{B}$ OBBLE-HEAD doll syndrome (BHDS) is a rare movement disorder first identified in the literature by Benton et al. ${ }^{5}$ The cardinal feature of BHDS is an involuntary 2- to $3-\mathrm{Hz}$ movement of the head in the anterior-posterior direction (as if nodding "yes-yes" like the "bobbing" of a bobble-head doll toy), in the lateral rotational direction (as if shaking the head "no-no"), or in some irregular alternating combination of the 2 directions.

We present 2 new cases of BHDS. The first has several unusual features, perhaps the most striking being the late age of symptom onset, a greater tendency toward the rarer lateral ("no-no") motions, and the patient's inability to voluntarily suppress the motions. The second is a more typical case, although the age of symptom onset is also unusually late.

A comprehensive review of the literature was per- formed by the first author (B.R.). A search for the term "bobble head" in both PubMed and MEDLINE was carried out. The cases from these reports were combined with those found in previous reviews. $17,19,23,28,33,44,48,49$ Various cases were then excluded: those lacking the cardinal head movement feature ${ }^{4,38,41}$ and those originally reported in publications (often non-English language) that we could not obtain. This yielded a total of 70 previous cases of BHDS. The list of cases extracted from the literature was not confirmed by any of the authors except first author. The list of previously published cases of BHDS combined with the 2 current cases, as well as a summary of their features, is compiled in Table 1.

The most recent BHDS review was published in 2005.19 This current updated review includes reports published since that time as well as other cases found in the literature (at times part of other series). Thus, in addition to pre-

ABBREVIATIONS BHDS = bobble-head doll syndrome; CVP = cystoventriculoperitoneal; ETV = endoscopic third ventriculostomy; VP = ventriculoperitoneal

SUBMITTED March 13, 2017. ACCEPTED September 6, 2017.

INCLUDE WHEN CITING Published online January 5, 2018; DOI: 10.3171/2017.9.PEDS16704. 
senting a new atypical case and another more typical one, we provide a comprehensive and up-to-date summary of BHDS as of the time of writing. We also obtained video documentation of the phenomenon, which will provide the clinician with an immediate impression of the clinical features of this condition.

\section{Case Reports Case 1}

History and Presentation

A 14-year-old boy presented with a 3-year history of headache with intermittent vomiting, recent cognitive decline, and a 6-week history of repetitive involuntary motions of the head and trunk that had increased in the past 3 days. Primary motion of the head was in a 2- to 3-Hz lateral-to-lateral rotation (as if shaking his head "no-no"), although some occasional anterior-to-posterior movements (as if nodding "yes-yes") were intermittently present as well. In addition, the patient would involuntarily flex and extend his trunk from time to time as if attempting to find a comfortable position in the bed. Involuntary head motions would stop whenever the patient would rest the posterior aspect of his head on the bed. Video 1 demonstrates head and truncal motions and gaze testing both before and after surgery.

VIDEO 1. Case 1 was a 14 -year-old boy with BHDS associated with a tectal mass. Primary head motions show a 2- to 3-Hz lateral-to-lateral ("no-no") rotation with occasional anterior-to-posterior ("yes-yes") movements. Involuntary truncal flexion and extension are present as well. Head motions stop when the patient rests the posterior aspect of his head on the bed. Eye movement testing shows some hesitation in upward gaze. Postsurgical excerpts show absence of involuntary motions and full eye movements. Copyright Bryan Renne. Published with permission. Click here to view.

\section{Examination}

On examination, the patient was conscious but slightly confused. He had macrocephaly $(60.5-\mathrm{cm}$ head circumference, which is more than 2 standard deviations above the age-sex mean) and pale optic discs. He was not able to stand or walk, so an assessment of gait was not feasible. He did show some lag in upward gaze but otherwise had no neurological deficits other than the repetitive motions of the head and trunk. An MRI study of the head showed a tectal mass causing aqueductal obstruction and obstructive triventriculomegaly (Fig. 1).

\section{Operation and Postoperative Course}

An endoscopic third ventriculostomy (ETV) was performed without complications. The patient's head and truncal motions resolved completely in the recovery room immediately after surgery. On 4-month follow-up, the motions had not returned. An MRI study obtained 9 months after surgery did show mild increased size of the tumor; however, the ventricles had decreased in size. Endocrinological follow-up has been unremarkable, though an adrenocorticotropic hormone (ACTH) stimulation test is scheduled. The patient will be followed up further by endocrinology and oncology departments.

\section{Case 2}

\section{History and Presentation}

A 7.5-year-old, left-handed girl presented with a 2-year history of recently worsening involuntary anterior-posterior bobbing motions of the head at approximately $3 \mathrm{~Hz}$. The movements were more severe when she was emotionally upset, would stop during sleep, and could be voluntarily suppressed for a couple of minutes. There was no discomfort or exhaustion associated with the head bobbing. A history of headache or vomiting was denied.

\section{Examination}

On examination, the patient was alert, oriented, and appeared healthy. She had normal cognitive development. Her head circumference was $53.5 \mathrm{~cm}$ (90th percentile). She had short stature $(110 \mathrm{~cm}$, which is more than 3 standard deviations below the age-sex mean). Eye movements were full, and there were no visual field deficits. However, bilateral optic atrophy was noted. Motor examination showed Grade 4+ proximal weakness of the upper and lower limbs, with normal strength distally. Reflexes were brisk, more so in the lower limbs (Grade 3+) than in the upper limbs (Grade 2+). Tandem gait was slightly unsteady both backward and forward. Plantar responses were normal, and clonus was absent. The sensory examination showed normal findings. An endocrine assessment revealed normal thyroid, cortisol, and antidiuretic hormone reserve; however, the patient did have growth hormone deficiency. She had a longstanding history of polyuria and polydipsia, but diabetes insipidus was ruled out. An MRI study of the head showed a large suprasellar cyst causing obstructive hydrocephalus (Fig. 2 and Video 2).

VIDEO 2. Case 2 was a 7.5-year-old girl with BHDS associated with a suprasellar cyst. Primary head motions are a $3-\mathrm{Hz}$ anteriorto-posterior ("yes-yes") movements. Gait testing is demonstrated. Intraoperative excerpt shows cyst fenestration and cautery.

Postsurgical excerpt shows absence of involuntary head motions.

Copyright Bryan Renne and Paul Steinbok. Published with permission. Click here to view.

\section{Operation and Postoperative Course}

An endoscopic fenestration of the cyst was performed. Cisternostomy was attempted but was aborted due to the occurrence of bradycardia. Because the cisternostomy could not be performed, the cyst wall was shrunk using cautery. The patient's head bobbing resolved in the recovery room immediately after surgery. However, intermittent anterior-posterior bobbing did return on the 2nd postoperative day, although it was greatly diminished compared with that seen at initial presentation and occurred only during times of fatigue or emotional upset. This intermittent head bobbing eventually ceased as the patient was followed up over a 7.5-year period. The patient was started on growth hormone shortly after surgery. Her growth over the follow-up period put her not far from the height expected based on her parents' heights. The patient was diagnosed at age 12 with autism spectrum disorder.

\section{Discussion}

The cardinal feature of BHDS is an involuntary 2- to 


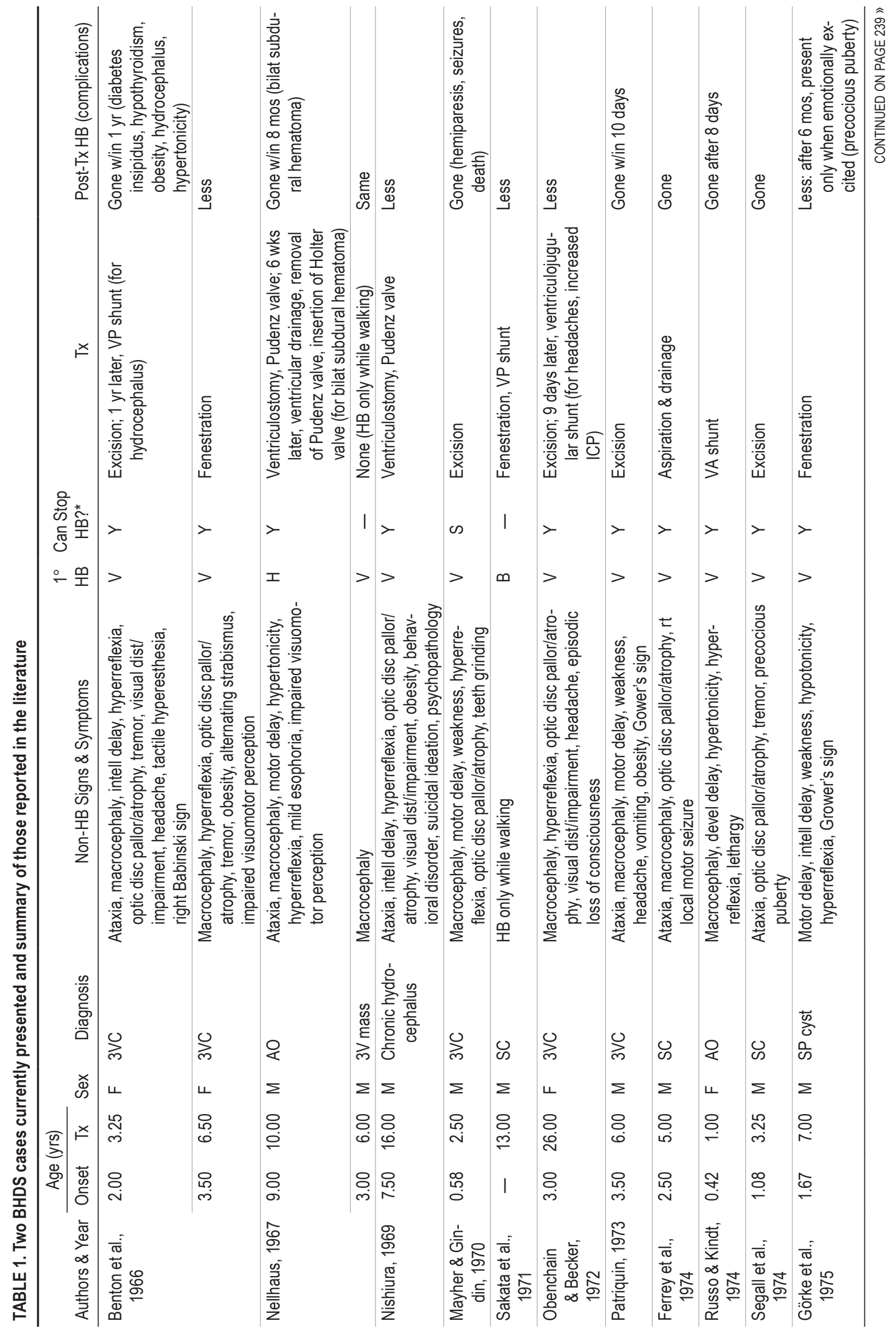




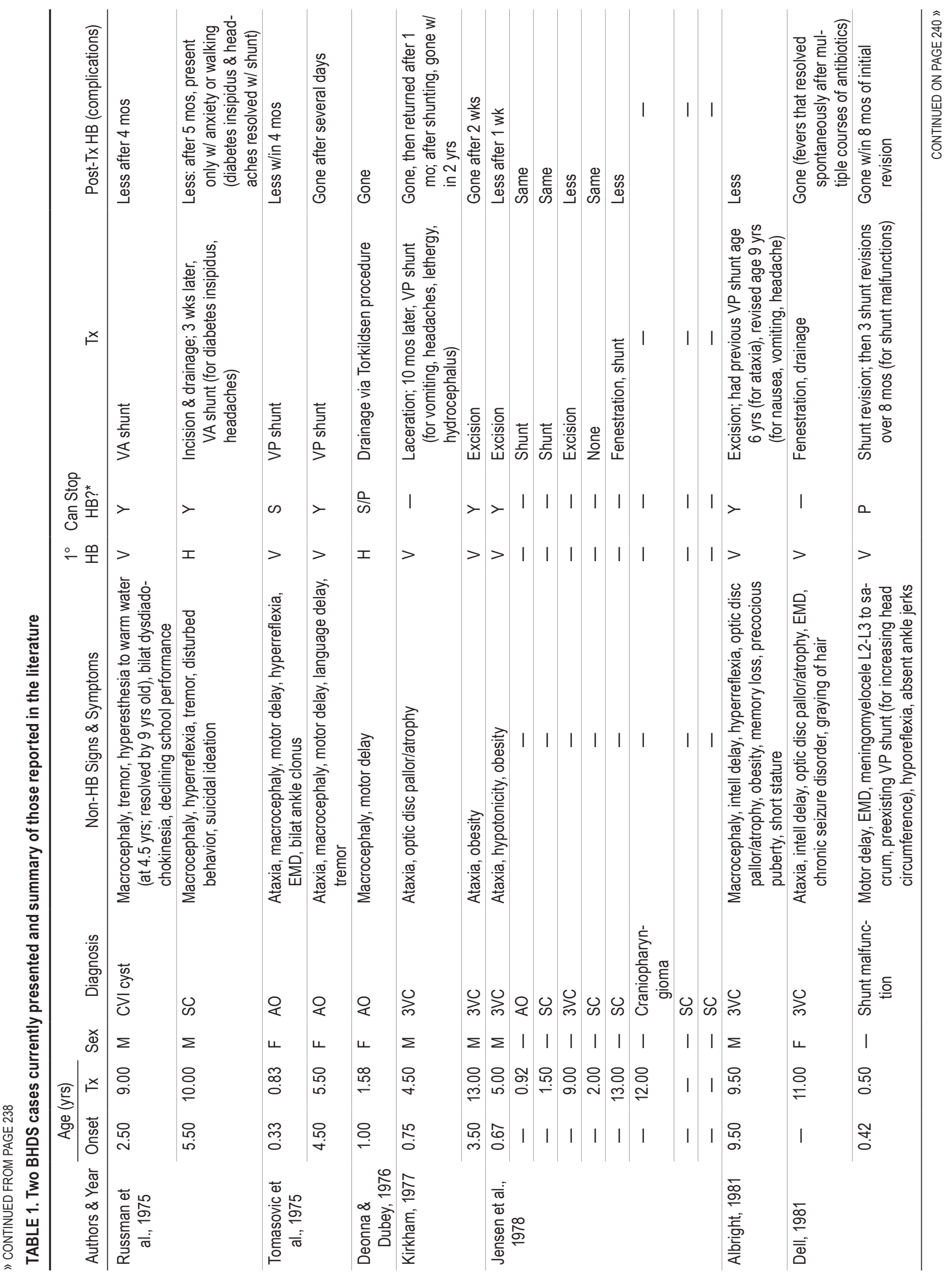




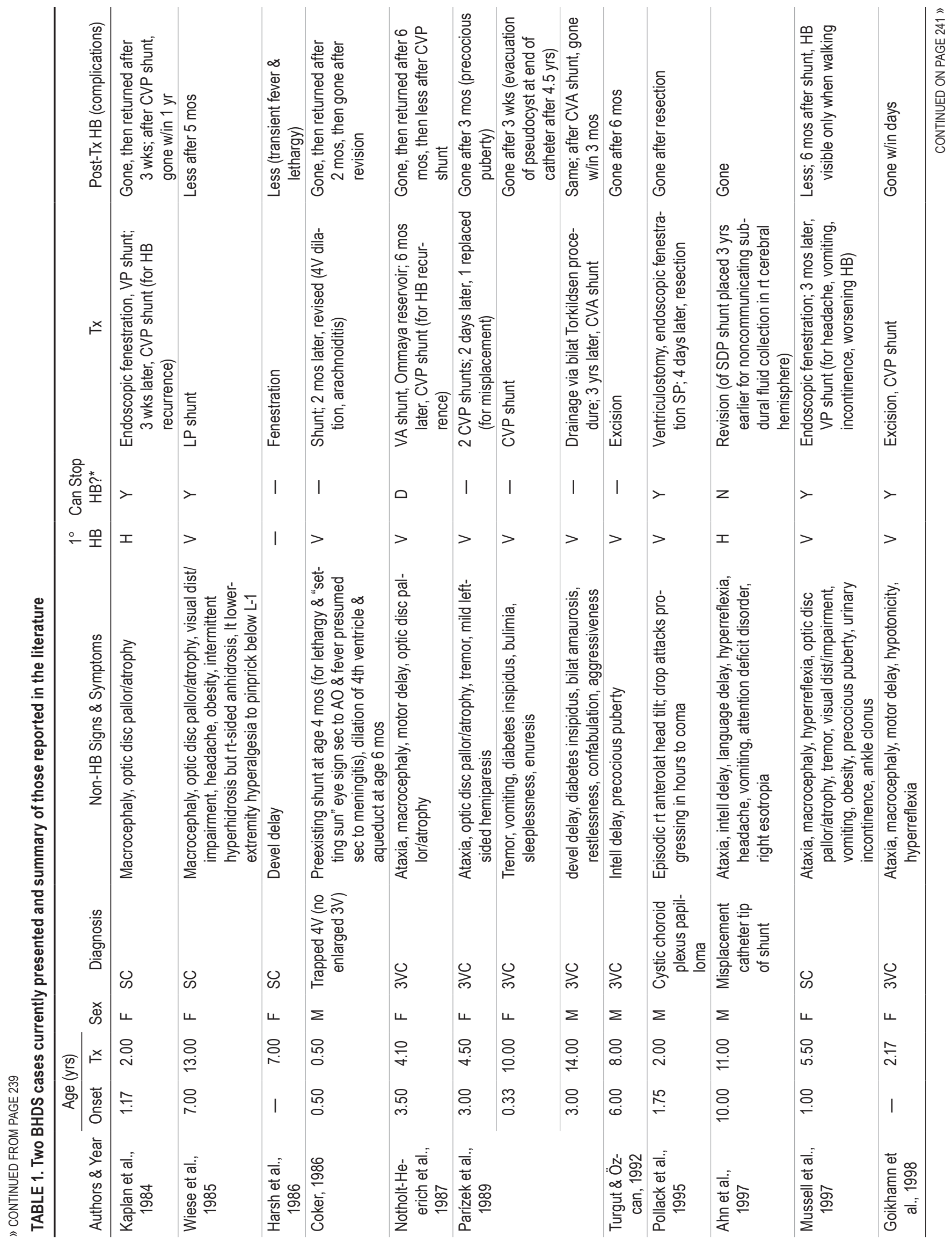




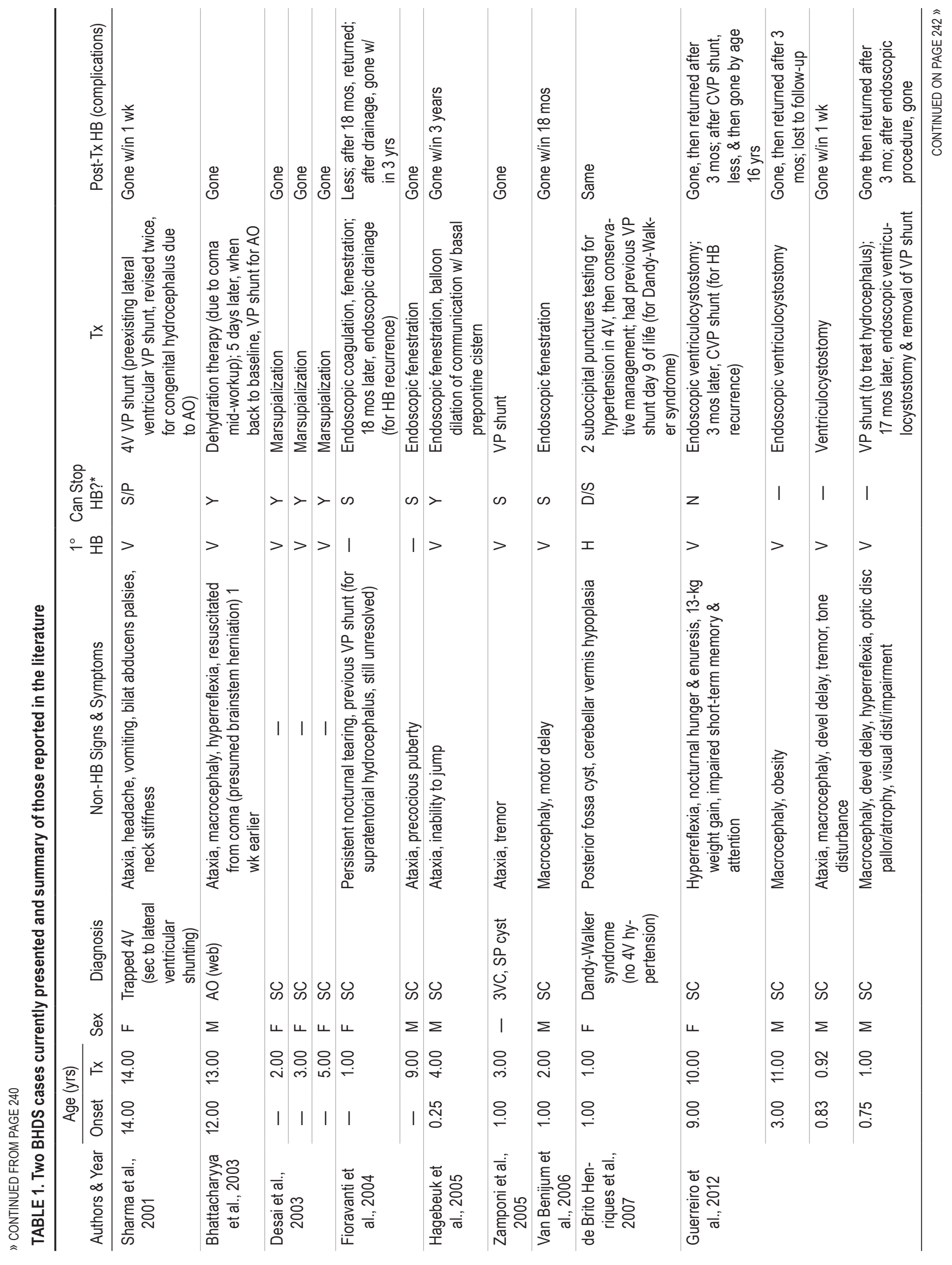




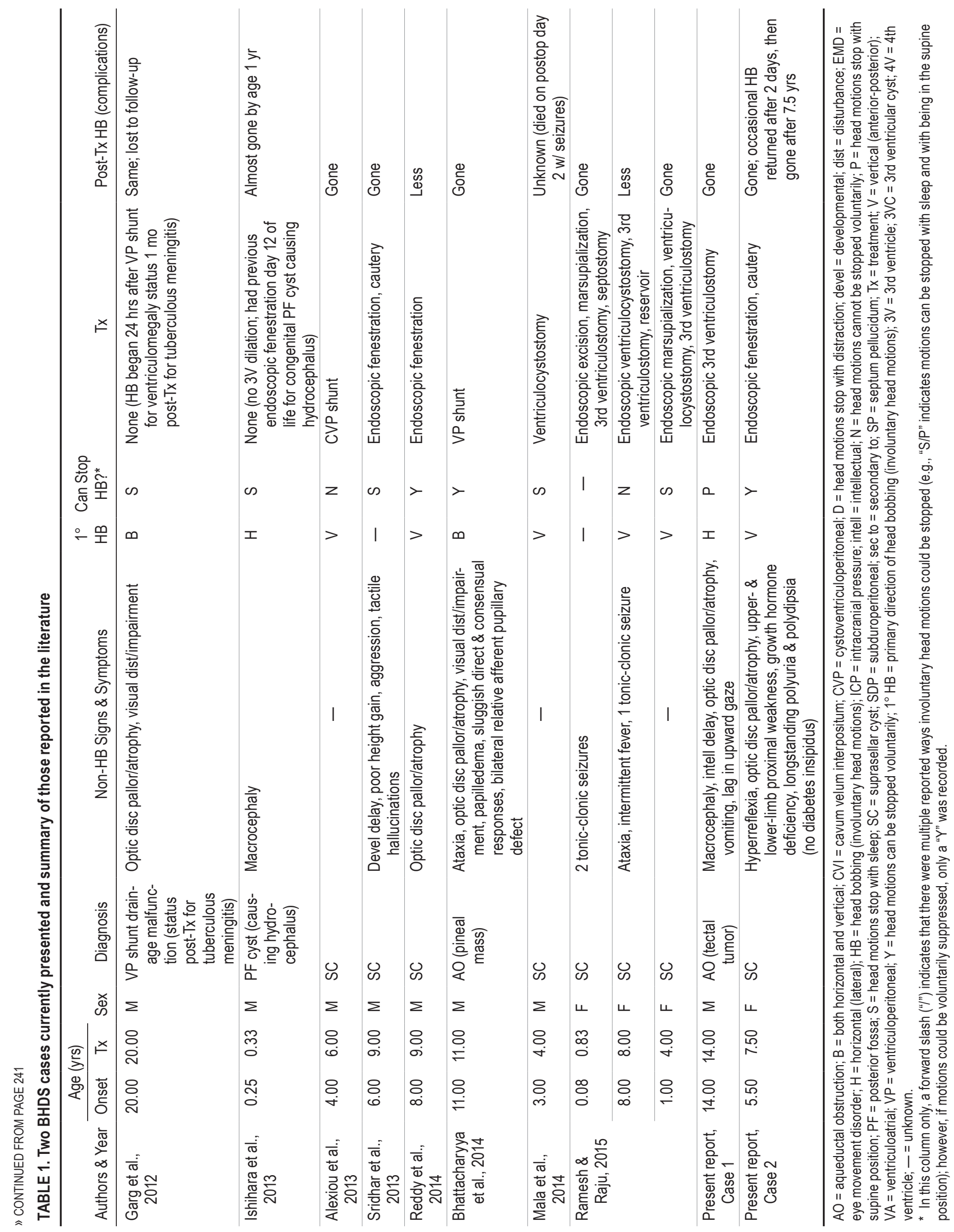



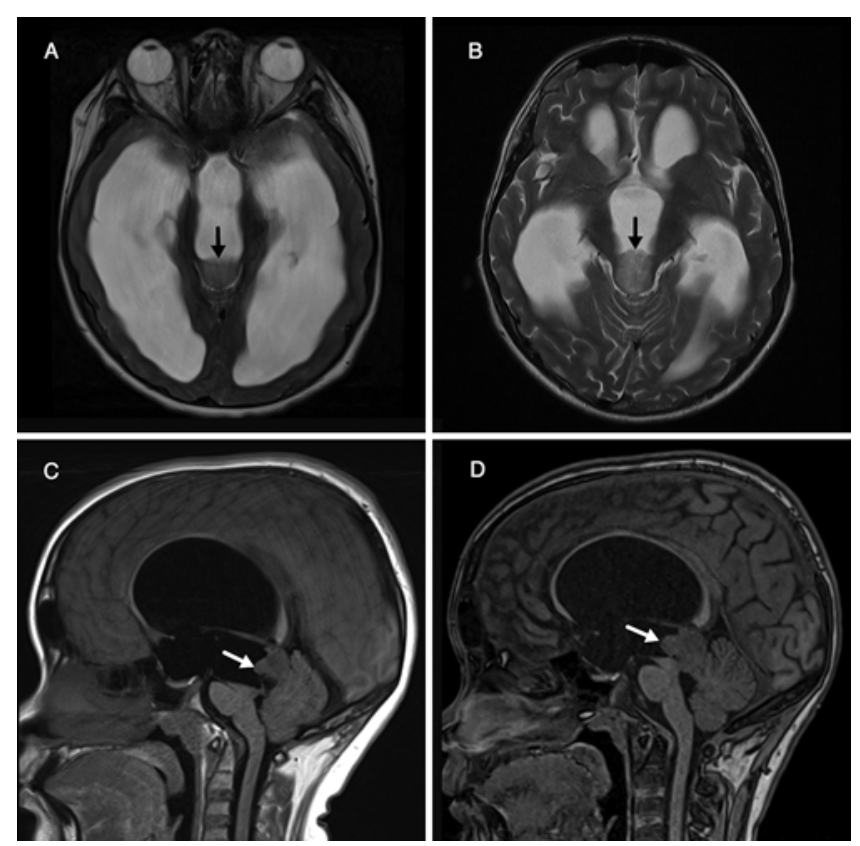

FIG. 1. Case 1. MR images obtained in a 14-year-old boy with BHDS associated with a tectal mass (white and black arrows). A: Presurgical axial T2-weighted image showing obstructive triventriculomegaly. B: Post-ETV axial T2-weighted image showing reduced size of the third and lateral ventricles. C: Presurgical sagittal turbo inversion recovery magnitude image showing aqueductal obstruction by tectal tumor causing triventricular dilation, bowing of the lamina terminalis and the floor of the third ventricle, and sellar erosion. D: Post-ETV sagittal magnetization-prepared rapid gradient-echo image showing a relaxed floor of the third ventricle. Post-ETV images were taken 9 months after the presurgical images and showed that the tectal mass increased in size to $19 \times 25 \times 24 \mathrm{~mm}^{3}$ (craniocaudal $\times$ anteroposterior $\times$ right-left) from a presurgical size of $19 \times 20 \times 20 \mathrm{~mm}^{3}$.

3-Hz movement of the head in the anterior-posterior ("yesyes") direction, the lateral ("no-no") rotational direction, or some irregular alternating combination of the 2 movements. In addition to involuntary head and truncal motions, information on presenting signs and symptoms was available in 58 of the 72 cases ( $81 \%$ of total, see Table 1). Common presenting signs and symptoms are summarized in Table 2. Other less common signs and symptoms not included in Table 2 are listed with individual cases in Table 1.

The age of onset of abnormal head movements was documented in 55 cases ( $76 \%$ of total), and in these cases the median onset age was 3.0 years (median absolute deviation of 3.35 years), with a range of 1 month to 20 years. Thus, BHDS is typically a disease of early childhood, and the onset age of 14 years in our Case 1 is the second oldest reported in the literature.

Of the 62 cases ( $86 \%$ of total) for which patient sex is identified, 35 were male (56\%) and 27 female (44\%) for a male/female ratio of 1.3:1. Previous reviews, which relied on a subset of the cases we have identified, report a higher male/female ratio of $2: 1$.

Of the 59 cases ( $82 \%$ of total) for which there was enough information to identify a direction of repetitive head movements, 48 were primarily anterior-posterior (i.e., $81 \%$ "yes-yes") and 8 were primarily lateral (i.e., $14 \%$ "no-no"). For patients with both anterior-posterior bob-
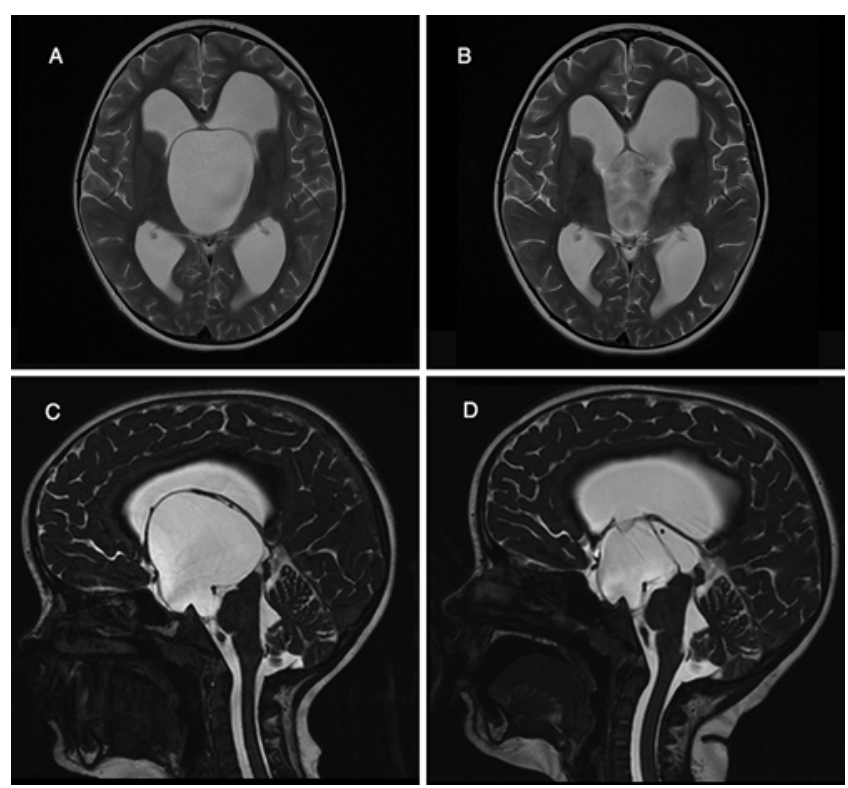

FIG. 2. Case 2. MR images acquired in a 7.5-year-old girl with BHDS associated with suprasellar cyst. Presurgical axial T2-weighted image (A) and presurgical sagittal constructive interference in steady-state (CISS) image $(C)$ showing a large suprasellar cyst. Post-fenestration axial T2-weighted image (B) and post-fenestration CISS image (D) showing reduced size of the cyst.

bing ("yes-yes") and lateral shaking ("no-no") movements, one direction took primacy over the other in all but 3 cases (5\%). The BHDS literature often mentions that head motions decrease or cease with sleep, distraction, or with patients in the supine position; increase with excitement or anxiety; and are voluntarily suppressible for a period of seconds to minutes. Regarding the increase or decrease in motion, the literature is often unclear as to whether this change refers to frequency, amplitude, perceived effort on the part of the patient, or something else. As for whether head motions are suppressible, of 50 cases $(69 \%$ of the total) in which this information was available, movements were reported as voluntarily suppressible for 29 patients (58\%); as not voluntarily suppressible for 4 (8\%); and as suppressible with one or more distraction, or sleep, or being in the supine position (and either not voluntarily suppressible or without mention of being voluntarily suppressible) in $17(34 \%)$. Of the 37 patients (51\% of total) treated at age 3 years or more with available data on whether head motions were suppressible, 24 patients $(65 \%)$ could suppress motions voluntarily, while 13 (35\%) either could not suppress head motions voluntarily or could do so only with one or more of distraction, or sleep, or being in the supine position. This suggests that repetitive head motions in BHDS can usually be suppressed, and, if the child is at least 3 years old at the time of treatment, suppression is most often voluntary. These considerations highlight 2 other rare features of our case 1: This 14-year-old patient could not suppress his head motions voluntarily (although he could do so when supine), and his primary direction of motion was lateral shaking ("no-no"). Our case 2 is more typical, in that the patient could temporarily suppress her head motions and the primary direction of motion was anterior-posterior bobbing ("yes-yes"). 
TABLE 2. Presenting signs and symptoms of BHDS

\begin{tabular}{lcc}
\hline \multicolumn{1}{c}{ Signs \& Symptoms } & $\begin{array}{c}\text { No. of Cases } \\
\text { (of 58) }\end{array}$ & $\begin{array}{c}\% \text { of } \\
\text { Cases }\end{array}$ \\
\hline Macrocephaly & 27 & 47 \\
\hline Ataxia & 25 & 43 \\
\hline $\begin{array}{l}\text { Impairment or developmental delay (cognition, } \\
\quad \text { motor, or language) }\end{array}$ & 24 & 41 \\
\hline Optic disc pallor or atrophy & 21 & 36 \\
\hline Hyperreflexia & 18 & 31 \\
\hline Tremor & 11 & 19 \\
\hline Obesity & 9 & 16 \\
\hline Endocrinopathy (including precocious puberty) & 9 & 16 \\
\hline Visual disturbance or impairment & 8 & 14 \\
\hline Headache & 6 & 10 \\
\hline Vomiting & 6 & 10 \\
\hline Weakness & 3 & 5 \\
\hline Hypotonicity & 3 & 5 \\
\hline Eye movement disorders & 3 & 5 \\
\hline Hypertonicity & 2 & 3 \\
\hline
\end{tabular}

Presenting signs and symptoms other than involuntary head motions were reported in 58 cases ( $81 \%$ of total).

In the 72 cases of this review, the major pathologies associated with BHDS were as follows:

- 32 cases (44\%): suprasellar cyst (including our Case 2). $3,12-14,18-20,23,24,26,28,36-38,40,41,43,47,48$

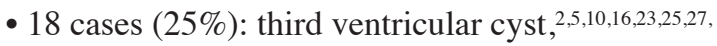
$31-34,46,49$ in one case concurrent with a septum pellucidum cyst. ${ }^{49}$

- 10 cases (14\%): aqueductal obstruction (aqueductal stenosis $8,11,23,29,39,44$ aqueductal web 1,7 pineal mass $1,{ }^{6}$ and tectal tumor 1 [our case 1]); 1 patient had an associated isolated trapped fourth ventricle. ${ }^{8}$

- 4 cases (6\%): shunt malfunction, misplacement, or complication, of which 2 were due to malfunction, ${ }^{1,10} 1$ was due to a trapped fourth ventricle after lateral ventricular shunting, ${ }^{42}$ and 1 was due to catheter placement obstructing the left foramen of Monro..$^{15}$

- 3 cases (4\%): other cysts impinging on the third ventricle, of which 1 was a septum pellucidum cyst, ${ }^{17} 1$ was a cyst of the cavum velum interpositum,,$^{38}$ and 1 was a cystic choroid plexus papilloma causing obstructive hydrocephalus. ${ }^{35}$

- 2 cases (3\%): masses impinging on the third ventricle, 1 a third ventricular mass ${ }^{29}$ and 1 a craniopharyngioma. ${ }^{23}$

- 2 cases (3\%): after fenestration of posterior fossa cysts, 1 after endoscopic fenestration but without enlargement of the third ventricle ${ }^{22}$ and 1 in a patient with
Dandy-Walker syndrome who did not have intracranial hypertension at the onset of head bobbing. ${ }^{9}$

- 1 case $(1 \%)$ : chronic hydrocephalus. ${ }^{30}$

The pathophysiology and causative anatomical substrate of BHDS is unknown, although various mechanisms have been suggested: distortion of structures or pathways adjacent to the third ventricle, perhaps involving the basal ganglia (since motions can often be voluntarily suppressed); ;,29,30 compression of the dorsomedial nucleus of thalamus or the dentatorubrothalamic pathway; ${ }^{19,39}$ a possible role of fetal viral infection in anterior horn cells; ${ }^{17}$ the mammillary-fornical-hippocampal circuit; ${ }^{2}$ a "learned behavior" causing transient relief of foraminal blockage or cystic volume reduction, ${ }^{35,48}$ which was demonstrated in one case using a CT cisternogram; ${ }^{48}$ pressure on the dorsomedial red nucleus; ${ }^{8}$ and cerebellar dysfunction, $, 9,22$ which has been noted to cause abnormal head motions. ${ }^{21,45}$

Treatment of BHDS is surgical, with the particular procedure depending on the associated pathology. Generally the treatment involves restoration of CSF flow and reducing pressure on compressed structures.

BHDS symptom recurrence has been reported in 8 cases (see Table 1), including our case 2:

- A patient with third ventricular cyst treated with laceration showed return of head motions within 1 month; a ventriculoperitoneal (VP) shunt ceased motions within 2 years..$^{25}$

- A patient with a suprasellar cyst treated with endoscopic fenestration and a VP shunt had return of head motions after 3 weeks; a cystoventriculoperitoneal (CVP) shunt ceased motions within 1 year. ${ }^{24}$

- A patient with trapped fourth ventricle treated by shunt placement had return of head motions after 2 months; these were eliminated after shunt revision. ${ }^{8}$

- A patient with obstructive hydrocephalus secondary to third ventricular cyst treated with a ventriculoatrial shunt and an Ommaya reservoir had head motion recurrence after 6 months; these were lessened but not eliminated after CVP shunt placement. ${ }^{31}$

- A patient with suprasellar cyst treated with endoscopic ventriculocystostomy had head motion recurrence after 3 months; a CVP shunt led to elimination of motions within a 16-year follow-up period. ${ }^{18}$

- A patient with a suprasellar cyst treated with endoscopic ventriculocystostomy had head motion recurrence after 3 months; the patient was subsequently lost to follow-up. ${ }^{18}$

- A patient with obstructive hydrocephalus secondary to a suprasellar cyst treated with VP shunting had head motion recurrence after 3 months; removal of the shunt and endoscopic ventriculocystostomy eliminated head motions. $^{18}$ 
- In our case 2, an SC was treated with endoscopic fenestration and cautery, and occasional head motions returned after 2 days, but head motions disappeared completely within the 7.5-year follow-up.

Thus, symptom recurrence in BHDS is not often reported (8 [11\%] of 72 cases reviewed), has occurred within 6 months of initial treatment in the cases known to us, and does not always require treatment. Nevertheless, given the possibility of growth of mass or recurrence of cyst, we elect to follow our BHDS patients with annual MRI. Assuming successful treatment and stable posttreatment clinical course, we transition to less frequent imaging as time progresses.

In conclusion, we have presented 2 new cases of BHDS and have performed a comprehensive review of the literature. We have also provided video documentation of the phenomenon, which will give the clinician an immediate impression of the clinical features of BHDS.

\section{References}

1. Ahn Y, Cho BK, Wang KC: Bobble-head doll syndrome associated with subduroperitoneal shunt malfunction. Childs Nerv Syst 13:234-237, 1997

2. Albright L: Treatment of bobble-head doll syndrome by transcallosal cystectomy. Neurosurgery 8:593-595, 1981

3. Alexiou GA, Sfakianos G, Prodromou N: Giant suprasellar arachnoid cyst with head bobbing. Mov Disord 28:1216, 2013

4. Banerjee T: Abnormal movements with hydrocephalus. Case report. J Neurosurg 46:674-676, 1977

5. Benton JW, Nellhaus G, Huttenlocher PR, Ojemann RG, Dodge PR: The bobble-head doll syndrome: report of a unique truncal tremor associated with third ventricular cyst and hydrocephalus in children. Neurology 16:725-729, 1966

6. Bhattacharyya KB, Deb S, Ghosh SN, Mondal S: Bobbling head in a young subject. Ann Indian Acad Neurol 17:371373, 2014

7. Bhattacharyya KB, Senapati A, Basu S, Bhattacharya S, Ghosh S: Bobble-head doll syndrome: some atypical features with a new lesion and review of the literature. Acta Neurol Scand 108:216-220, 2003

8. Coker SB: Bobble-head doll syndrome due to trapped fourth ventricle and aqueduct. Pediatr Neurol 2:115-116, 1986

9. de Brito Henriques JG, Henriques KSW, Filho GP, Fonseca LF, Cardoso F, Da Silva MC: Bobble-head doll syndrome associated with Dandy-Walker syndrome. Case report. J Neurosurg 107 (3 Suppl):248-250, 2007

10. Dell S: Further observations on the "bobble-headed doll syndrome". J Neurol Neurosurg Psychiatry 44:1046-1049, 1981

11. Deonna T, Dubey B: Bobble-head doll syndrome. Case report with a review on the different types of abnormal head movements in infancy, and their clinical significance. Helv Paediatr Acta 31:221-227, 1976

12. Desai KI, Nadkarni TD, Muzumdar D, Goel A: Suprasellar arachnoid cyst presenting with bobble-head doll movements: a report of 3 cases. Neurol India 51:407-409, 2003

13. Ferrey PC, Feldman V, Kerber C: The bobble-head doll syndrome due to a basal arachnoid cyst. Neurology 24:394, 1974

14. Fioravanti A, Godano U, Consales A, Mascari C, Calbucci F: Bobble-head doll syndrome due to a suprasellar arachnoid cyst: endoscopic treatment in two cases. Childs Nerv Syst 20:770-773, 2004

15. Garg RK, Singh SK, Malhotra HS, Singh MK: Abnormal head movement in a patient with tuberculous meningitis. BMJ Case Rep 2012:bcr2012006663, 2012

16. Goikhman I, Zelnik N, Peled N, Michowiz S: Bobble-head doll syndrome: a surgically treatable condition manifested as a rare movement disorder. Mov Disord 13:192-194, 1998

17. Görke W, Pendl G, Pandel C: Spinal muscular atrophy in a boy with head-nodding resulting from a large septum pellucidum cyst. Neuropediatrics 6:190-201, 1975

18. Guerreiro H, Vlasak A, Horinek D, Tichy M, Lisy J, Vanek P, et al: Bobble-head doll syndrome: therapeutic outcome and long-term follow-up in four children. Acta Neurochir (Wien) 154:2043-2049, 2012

19. Hagebeuk EEO, Kloet A, Grotenhuis JA, Peeters EAJ: Bobble-head doll syndrome successfully treated with an endoscopic ventriculocystocisternostomy. Case report and review of the literature. J Neurosurg 103 (3 Suppl):253-259, 2005

20. Harsh GR IV, Edwards MSB, Wilson CB: Intracranial arachnoid cysts in children. J Neurosurg 64:835-842, 1986

21. Hottinger-Blanc PMZ, Ziegler AL, Deonna T: A special type of head stereotypies in children with developmental (?cerebellar) disorder: description of 8 cases and literature review. Eur J Paediatr Neurol 6:143-152, 2002

22. Ishihara M, Nonaka M, Oshida N, Hamada Y, Nakajima S, Yamasaki M: "No-no" type bobble-head doll syndrome in an infant with an arachnoid cyst of the posterior fossa: a case report. Pediatr Neurol 49:474-476, 2013

23. Jensen HP, Pendl G, Goerke W: Head bobbing in a patient with a cyst of the third ventricle. Childs Brain 4:235-241, 1978

24. Kaplan BJ, Mickle JP, Parkhurst R: Cystoperitoneal shunting for congenital arachnoid cysts. Childs Brain 11:304-311, 1984

25. Kirkham TH: Optic atrophy in the bobble-head doll syndrome. J Pediatr Ophthalmol 14:299-301, 1977

26. Mala R, Lakshmi AM, Nagireddy NB, Chiniga VR: Suprasellar arachnoid cyst presenting with Bobble-head doll syndrome. J NTR Univ Health Sci 3:48-50, 2014

27. Mayher WE III, Gindin RA: Head bobbing associated with third ventricular cyst. Report of a case. Arch Neurol 23:274-277, 1970

28. Mussell HG, Dure LS, Percy AK, Grabb PS: Bobble-head doll syndrome: report of a case and review of the literature. Mov Disord 12:810-814, 1997

29. Nellhaus G: The bobble-head doll syndrome: a "tic" with a neuropathologic basis. Pediatrics 40:250-253, 1967

30. Nishiura N: A case of bobble-head doll syndrome associated with psychopathic behaviors. Folia Psychiatr Neurol Jpn 23:275-283, 1969

31. Notholt-Heerich B, Körholz D, Voit T, Lumenta C: Das Bobble head doll Syndrom. Klin Padiatr 199:77-79, 1987

32. Obenchain TG, Becker DP: Head bobbing associated with a cyst of the third ventricle. Case report. J Neurosurg 37:457-459, 1972

33. Parízek J, Nĕmecková J, Šercl M: Bobble-head doll syndrome associated with the III ventricular cyst. Three cases in children 7 years after CVP or CVA shunting. Childs Nerv Syst 5:241-245, 1989

34. Patriquin HB: The bobble-head doll syndrome. A curable entity. Radiology 107:171-172, 1973

35. Pollack IF, Schor NF, Martinez AJ, Towbin R: Bobble-head doll syndrome and drop attacks in a child with a cystic choroid plexus papilloma of the third ventricle. Case report. J Neurosurg 83:729-732, 1995

36. Ramesh S, Raju S: Suprasellar arachnoid cyst presenting with bobble-head doll syndrome: report of three cases. J Pediatr Neurosci 10:18-21, 2015

37. Reddy OJ, Gafoor JA, Suresh B, Prasad PO: Bobble head doll syndrome: a rare case report. J Pediatr Neurosci 9:175-177, 2014 
38. Russman BS, Tucker SH, Schut L: Slow tremor and macrocephaly: expanded version of the bobble-head doll syndrome. J Pediatr 87:63-66, 1975

39. Russo RH, Kindt GW: A neuroanatomical basis for the bobble-head doll syndrome. J Neurosurg 41:720-723, 1974

40. Sakata K, Yamada H, Yamamoto M, Takahashi C: [Bobblehead doll syndrome: report of a case with a large basal arachnoid cyst.] Brain Nerve 23:693-700, 1971 (Jpn)

41. Segall HD, Hassan G, Ling SM, Carton C: Suprasellar cysts associated with isosexual precocious puberty. Radiology 111:607-616, 1974

42. Sharma RR, Pawar SJ, Devadas RV, Dev EJ: CT stereotaxy guided lateral trans-cerebellar programmable fourth ventriculo-peritoneal shunting for symptomatic trapped fourth ventricle. Clin Neurol Neurosurg 103:143-146, 2001

43. Sridhar S, Purkayastha M, Dutta P, Dhandapani SS, Mukherjee KK: Third ventricular cyst presenting with bobble head doll movements and tactile hallucinations. Neurol India 61:87-88, 2013 (Letter)

44. Tomasovic JA, Nellhaus G, Moe PG: The bobble-head doll syndrome: an early sign of hydrocephalus. Two new cases and a review of the literature. Dev Med Child Neurol 17:777-783, 1975

45. Tully HM, Dempsey JC, Ishak GE, Adam MP, Mink JW, Dobyns WB, et al: Persistent figure-eight and side-to-side head shaking is a marker for rhombencephalosynapsis. Mov Disord 28:2019-2023, 2013

46. Turgut M, Özcan OE: Suprasellar arachnoid cyst as a cause of precocious puberty and bobble-head doll phenomenon. Eur J Pediatr 151:76, 1992

47. Van Beijnum J, Hanlo PW, Han KS, Ludo Van der Pol W, Verdaasdonk RM, Van Nieuwenhuizen O: Navigated laserassisted endoscopic fenestration of a suprasellar arachnoid cyst in a 2-year-old child with bobble-head doll syndrome. Case report. J Neurosurg 104 (5 Suppl):348-351, 2006
48. Wiese JA, Gentry LR, Menezes AH: Bobble-head doll syndrome: review of the pathophysiology and CSF dynamics. Pediatr Neurol 1:361-366, 1985

49. Zamponi N, Rychlicki F, Trignani R, Polonara G, Ruggiero $\mathrm{M}$, Cesaroni E: Bobble head doll syndrome in a child with a third ventricular cyst and hydrocephalus. Childs Nerv Syst 21:350-354, 2005

\section{Disclosures}

The authors report no conflict of interest concerning the materials or methods used in this study or the findings specified in this paper.

\section{Author Contributions}

Conception and design: Renne, Rueckriegel, Steinbok, Singhal. Acquisition of data: all authors. Analysis and interpretation of data: Renne, Rueckriegel, Radic, Steinbok, Singhal. Drafting the article: Renne, Steinbok, Singhal. Critically revising the article: all authors. Reviewed submitted version of manuscript: all authors. Approved the final version of the manuscript on behalf of all authors: Renne. Study supervision: Singhal.

\section{Supplemental Information \\ Videos}

Video 1. https://vimeo.com/240166777.

Video 2. https://vimeo.com/240166907.

\section{Correspondence}

Bryan Renne: BC Children's Hospital, Vancouver, BC, Canada. bryan@renne.org. 